\title{
End Image Defect Detection of Float Glass Based on Faster Region-Based Convolutional Neural Network
}

\author{
Dabing Jin ${ }^{1,2}$, Shiqing $\mathrm{Xu}^{1}$, Lianjie Tong ${ }^{1}$, Linyu $\mathrm{Wu}^{3}$, Shimin $\mathrm{Liu}^{1 *}$ \\ ${ }^{1}$ State Key Laboratory of Metastable Materials Science and Technology, Yanshan University, Qinhuangdao 066004, China \\ ${ }^{2}$ North China Institute of Aerospace Engineering, Langfang 065000, China \\ ${ }^{3}$ Hebei CSG Glass Co., Ltd., 28 Baihe Road Yongqing Industrial Park, Langfang 065600, China
}

Corresponding Author Email: 1sm@ysu.edu.cn

https://doi.org/10.18280/ts.370513

Received: 8 May 2020

Accepted: 3 September 2020

\section{Keywords:}

float glass, defect detection, faster regionbased convolutional neural network (Faster $R C N N)$, target detection, end image

\begin{abstract}
The float glass contains various defects for reasons of raw materials and production process. These defects can be observed on the end images of the glass. Since the defects are correlated with specific links of the production process, it is possible to discover the process problems by identifying the location and type of defects in end images. Based on faster region-based convolutional neural network (Faster RCNN), this paper proposes a deep learning method that improves the feature extraction network, and adds a Laplacian convolutional layer to preprocess the end images. Considering the defect features in end images, the anchor box size was adjusted to speed up the training. Besides, the lack of generalizability induced by small dataset was solved through data enhancement. With improved VGG16 as the feature extraction layer, a glass defect detection model was established, whose generalizability was improved through transfer learning. The experimental results show that the proposed model achieved a mean detection accuracy of $94 \%$ on actual test set, meeting the requirements for actual use in factories.
\end{abstract}

\section{INTRODUCTION}

The quality of float glass is directly related to the production process $[1,2]$. If there is a problem with the glass quality, the normal countermeasure is to ask experts to analyze the end images and production technique, and find an empirical solution. However, only the engineers and technicians with rich experience in glass production can diagnose glass problems [3]. Some problems even require repeated experiments to adjust the links of the production process. Any process adjustment will consume lots of materials, and bring a heavy burden to the manufacturer. In addition, the manual diagnosis tends to be highly subjective. Different experts may come up with different conclusions on the same phenomenon.

To guide glass production and save economic cost, it is of high necessity to realize the automatic diagnosis of problems in glass production. A possible solution is to learn the massive data on end images and the corresponding technical problems through deep learning, and then disclose the correlations between image features and the links of the production process. However, there is no report that applies deep learning to such problems. Therefore, the basic idea of this paper is as follows: With the aid of machine learning, design an algorithm to automatically learn the massive data on production process, as well as the corresponding end images, of the manufacturer on the computer, and reveal the hidden laws in the massive data. These laws will guide the glass production and end the dependence on human experts.

This paper applies the faster region-based convolutional neural network (Faster RCNN) to glass image defect detection. First, the number of data samples was expanded through image enhancement, and a Laplacian convolutional layer was added before the input layer of the Faster RCNN to sharpen the images, highlighting the texture features. Besides, the VGG16 model was used as the feature extraction network. Considering the defect features of glass images, the original anchor box size was adjusted to improve training and detection speeds. Moreover, the generalizability of our model was enhanced through transfer learning.

\section{LITERATURE REVIEW}

There are already many methods for defect detection through computer recognition of images. The conventional image processing algorithms are usually adopted to detect the defects of glass images, namely, grayscale-based image segmentation and shape-based defect detection. These conventional algorithms first establish a feature model through manual analysis on glass defect features, and then identify these features through image processing. The feature extraction and recognition completely depend on the image processing algorithm. The universality is severely lacking, for one algorithm only applies to one kind of features. As a result, the conventional algorithms cannot satisfactorily detect the various and combinatory defects in glass images.

In recent years, machine learning has given rise to many classification methods, such as support vector machine (SVM), and random forest (RF). These methods extract features by conventional algorithms, and classify the extracted features through machine learning. The machine learning improves the performance and accuracy of classification to a certain extent. Nevertheless, these new classification methods fail to achieve an ideal effect in actual production, because the sliding 
window method, which is adopted by them to locate the target, has a high time complexity and computing load.

The boom of deep learning has brought many academic achievements. In 2006, Hinton G. proposed a layer-by-layer greedy algorithm, setting off an upsurge in the development of artificial intelligence. Bengio and Lencun created the convolution neural network $(\mathrm{CNN})$, which has been successfully applied in digital image processing and widely used in computer vision. Since then, many networks have been derived from the $\mathrm{CNN}$ for image classification, image segmentation, and target detection. In 2014, Grischichk applied deep learning to target detection, and put forward the $\mathrm{R}-\mathrm{CNN}$, which combines region proposal with $\mathrm{CNN}$, relies on SVM for classification, and locates target with linear regression. In 2015, Girshick added spatial pyramid pooling (SPP) to R-CNN, and proposed the Faster-RCNN model, which greatly shortens the training time. In the same year, Grishick and He designed a Faster-RCNN capable of end-toend training through an additional region proposal network (RPN) [4].

\section{GLASS DEFECT DETECTION BASED ON FASTER- RCNN}

In essence, the detection of glass defects is to determine the type and location of each defect [5]. This task can be solved effectively through target detection in computer vision. With the development and application of deep learning, many target detection algorithms have emerged. The mainstream target detection algorithms: Region proposal-based R-CNN algorithms, and one-stage algorithms like you look only once (YOLO) and single shot detector (SSD) [6].

The R-CNN algorithms need to generate the candidate area, i.e. target position, and then classify and regress the candidate boxes. The accuracy of these algorithms has been slowly improving through the evolution from R-CNN, Fast R-CNN, to Faster R-CNN. But the high accuracy of these algorithms comes at the cost of computing speed. The one-stage algorithms can rapidly predict the type and location of different targets, using only one CNN. But the prediction accuracy is rather low [7].

In this paper, computing speed is not a priority, because glass defect detection does not require real-time detection on the production line. The detection accuracy is relatively important, as it directly bears on process adjustment $[8,9]$. Each process adjustment needs a lot of resources. Therefore, this paper adopts the highly accurate Faster R-CNN algorithm to detect glass defects.

Considering the defect features of end images, this paper improves the original Faster R-CNN: A Laplacian convolutional layer was added after the input layer to enhance the texture features and speed up training convergence; the VGG16 network was taken as the feature extraction layer [10], and the last fully connected layer was removed; the original anchor box size was modified such that the box attaches greater importance to large features and obvious defects than details. In addition, the training data were expanded through data enhancement, to increase the labeled data in the original dataset.

\subsection{Data enhancement}

The deep CNN has a huge amount of training parameters.
The training demand cannot be satisfied without lots of labeled data [11]. However, it is very laborious to label the data on end images: The glass images need to be collected in actual production, and then the location and type of defects need to be judged manually and labeled in turn.

This paper expands the small labeled dataset through data enhancement. The common methods for data enhancement include cropping, rotation, color change, brightness change, and contrast change [12]. The location of a glass defect, which is related to the link of the production process that leads to the defect, is a major feature of the defect. Thus, the data enhancement should not change the position of any pixel. Here, the data are enhanced by changing brightness and contrast.

\subsection{Laplacian convolutional layer}

The end images generally present a striped form. Sharpening the image can provide the grayscale difference of edge pixels, making the texture boundary clearer and the features more obvious. During image sharpening, the difference operation can reflect the grayscale of each pixel [13], determine every edge pixel, and enhance its pixel value. The common image sharpening methods include gradient method, Roberts operator method [14], Laplacian operator method [15], high-pass filtering, and template matching [16].

Considering the merits and defects of various sharpening methods, this paper adds convolutional sharpening as a network layer behind the output layer, with the aim to sharpen the input image at a low cost. With Laplacian operator as the convolution kernel [17], the added layer does not alter the size of the output image.

Laplacian algorithm is a linear quadratic differential operator with rotation invariance. This algorithm can sharpen image edges in different directions, and output results with refined boundaries and rich details. As a typical second-order derivative operator, Laplacian operator enhances the discontinuity of the gray value in the image, and reduces the gradual change of the gray area. The gradient of image $f(x, y)$ can be described as [18]:

$$
\mathrm{G}(f(x, y))=\left[\frac{\partial f}{\partial x}, \frac{\partial f}{\partial y}\right]^{T}
$$

In formula (1), the original image gradient is a vector, pointing to the direction with the greatest change rate of function $f(x, y)$. In this paper, the four-adjacent Laplacian operator is used, whose value depends on the gray difference of four adjacent pixels. The Laplacian operation can be achieved through template convolution [19] (Figure 1).

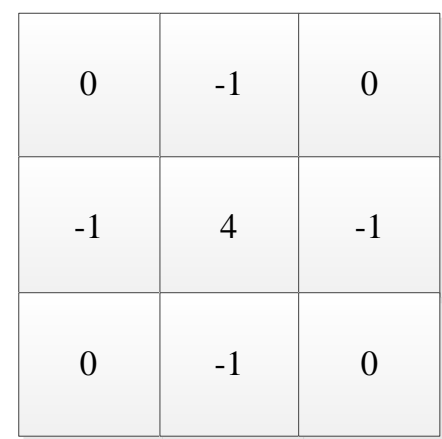

Figure 1. The Laplacian convolution kernel 
The four-adjacent Laplacian operator was taken as the convolution kernel to construct a layer of Laplacian convolutional layer [20] that processes the input image. While retaining the original information of the image, the added layer enhances the contrast on the edges with suddenly changing grayscale. When applied to glass images, the added layer helps to highlight the contour of defect, making the blurred image clearer and easier to detect.

\subsection{Faster-RCNN}

Based on R-CNN and Fast RCNN, Ross B. Girshick put forward the Faster RCNN in 2016 [21]. In network structure, Faster RCNN integrates feature extraction, proposal extraction, bounding box regression, and classification into one network, and realizes end-to-end training. The Faster RCNN greatly outperforms the R-CNN and Fast RCNN, especially in detection speed. As shown in Figure 2, Faster-RCNN can be divided into four parts:

(1) Feature extraction layer

Faster RCNN produces a feature map through a series of convolutions, activations, and pooling operations, and shares the feature map with the subsequent RPN layer and fully connected layer [22].

(2) RPN

The PRN, which produces region proposals, is the biggest difference between Faster and Fast RCNNs. Specifically designed for recommending regions of interest (ROIs), the RPN can be understood as a full convolutional network (FCN) that supports end-to-end training. In this layer, the softmax judges whether an anchor is positive or negative, and obtains an accurate proposal by modifying the anchors through bounding box regression.

(3) ROI pooling layer

This layer extracts the proposal feature maps from the feature maps and the proposals generated by the RPN. The ROIs are mapped into the feature maps, and pooled into regional feature maps with the same size [23]. Then, the regional feature maps are forwarded to the subsequent fully connected layer for target classification.

(4) Fully connected layer

This layer calculates the class of each proposal based on proposal feature maps [24], and determines the final location of the detection box through another bounding box regression.

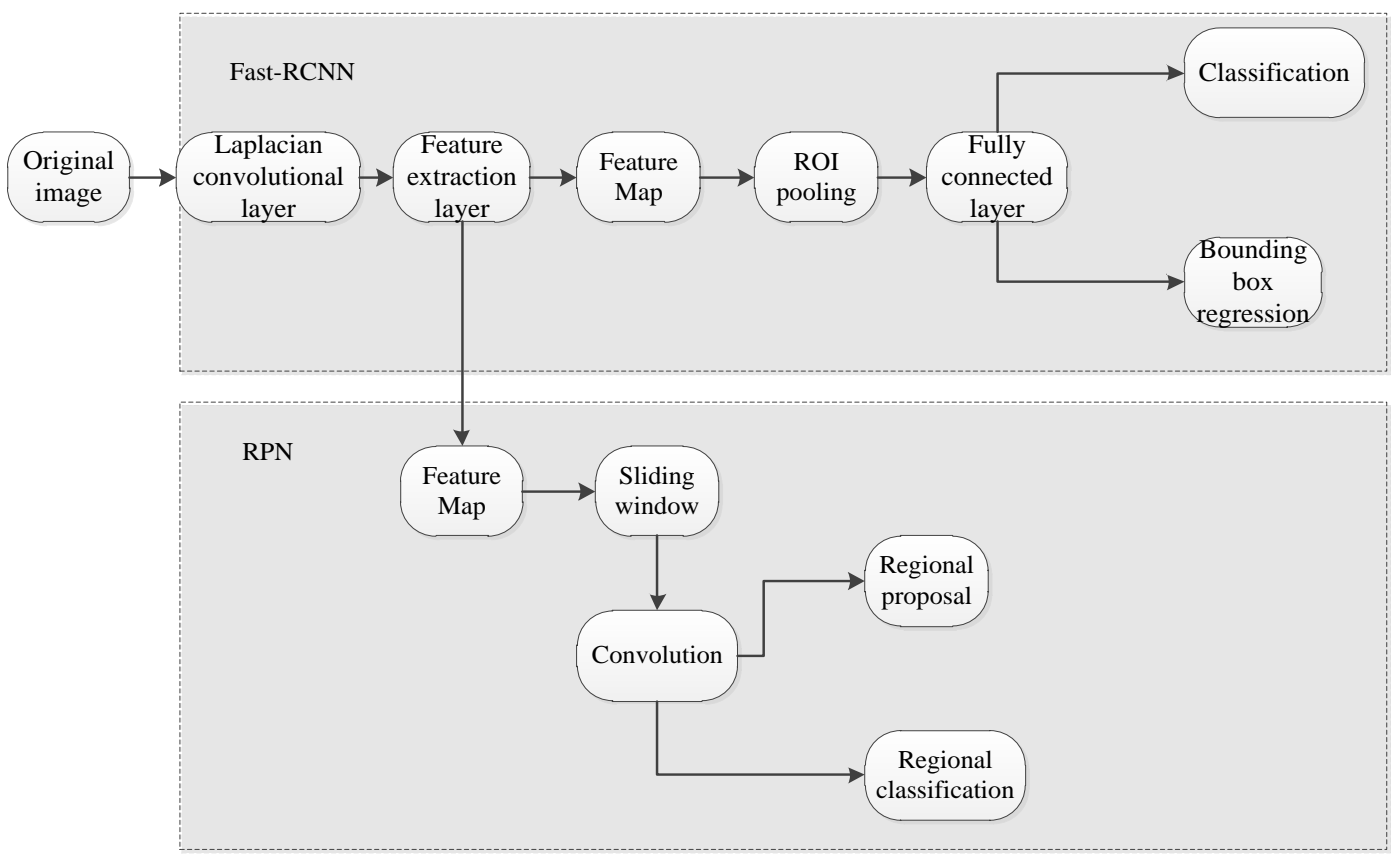

Figure 2. The structure of Faster R-CNN

\subsection{Improvement of Faster RCNN}

(1) Improved feature extraction layer

To detect the defect features of end images, the feature extraction layer must inherit the merits of deep networks in extracting high-level abstract features, while reducing the model complexity and training time. Since the glass defect detection is a binary classification problem, it is desirable to adopt the VGG16 network.

The VGG16 network includes 13 convolutional layers, 13 activation layers, 4 pooling layers, and a fully connected layer. In actual training, the image edges were supplemented through convolution, using a $3 * 3$ kernel, padding $=1$, stride $=1$, pooling $=2 * 2$, and stride $=2$. This simplifies the computing process.

Image textures are less complex than the features of other objects. It is sufficient to directly use VGG16 network for feature extraction. However, the complex structure of VGG will incur additional computing cost, increasing the risk of overfitting. Moreover, the parameters of the fully connected layer in the VGG account for a large portion of network parameters [25]. To simplify the network, the last two fully connected layers were replaced with a fully connected layer of 1,024 nodes. In addition, transfer learning was adopted to use the weight of ImageNet, thereby overcoming overfitting.

(2) Anchor box size

Anchors can be understood as some boxes of preset size. Let $\mathrm{k}$ be the number of types of anchors [26]. In the original network, $\mathrm{k}=9$, i.e. the anchor boxes have three different areas and aspect ratios. The anchor size was selected as per the specific target image.

To train the RPN, the first step is to generate anchor boxes. In the original network, anchor boxes of three different aspect ratios were generated with each pixel as the center. In total, 
nine kinds of anchor boxes were produced, whose aspect ratios are $0.5,1$, and 2 , and sizes are 8,16 , and 32 .

Using the original anchor boxes, the receptive field corresponding to each pixel of all feature maps is $16^{*} 16$ in size after pooling. By the smallest scale mapping, the anchor box size fell between 128 and 512. The anchor box size should be increased to suit the relatively large scale of glass defects. Therefore, the anchor box sizes were changed into 8, 16, and 32 . Finally, the anchor box areas were defined as $256 * 256$, $512 * 512$, and $1,024 * 1,024$, which are in line with the scales of glass defects.

Each anchor box thus generated was trained with positive and negative samples selected by intersection over union (IoU). The IoU values are as shown in Table 1.

Table 1. The IoU values for anchor boxes

\begin{tabular}{cc}
\hline Class & Criteria \\
\hline Positive & $\begin{array}{c}\text { IOU }>0.7 \text { or the IoU between anchor box } \\
\text { and target box maximized } \\
\text { IOU }<0.3\end{array}$ \\
$\begin{array}{c}\text { Negative } \\
\text { Others }\end{array}$ & $\begin{array}{c}\text { Image boundaries and other discarded } \\
\text { samples not for training }\end{array}$ \\
\hline
\end{tabular}

By reducing the number of anchors and increasing the area of the anchor box, the authors reduced the computing load and increased the training speed. In addition, some minor defects were neglected, allowing the model to focus on the defects with more obvious features and large coverage. In fact, only these features can accurately reflect the features of the production process.

(3) Adding Laplacian convolutional layer

A Laplacian convolutional layer was added before the Faster RCNN. Like other convolutional layers, the Laplacian convolutional layer aims to extract image features of different scales, except that the parameters of this layer are fixed, without needing to participate in training. This layer enhances the defect features in end images, and speeds up network convergence in training.

\section{EXPERIMENTS AND RESULT ANALYSIS}

First, the glass end images were collected on the production line by a float glass streak instrument. Under expert guidance, the type and location of glass defects were labeled, and the technical reasons and solutions of these defects were provided. Then, the defect data were organized into training sets to train our detection model. The trained model was tested on a test set. The effectiveness of the model was evaluated by mean accuracy, mean loss, and mean error. In addition, the model was compared with the original Faster RCNN.

\subsection{Sample production}

The experimental data were collected from major float glass manufacturers. The raw data, consisting of 1,200 images, contain the end images, and an XML file of defect labels and reasons. 800 images were allocated by random to a training set, and 400 to a test set in an independent manner (Table 2).

Table 2. The sample number and scale of datasets

\begin{tabular}{ccc}
\hline Datasets & Sample number & Minimum resolution \\
\hline Training set A & 800 & $4,200 \times 768$ \\
Training set B & 3,200 & $4,200 \times 768$ \\
Test set C & 400 & $4,200 \times 768$ \\
\hline
\end{tabular}

As shown in Table 2, training set A contains original data, while training set $\mathrm{B}$ adopts enhanced data. The data enhancement was realized by changing contrast and brightness, creating 3,200 training images for set $\mathrm{B}$. The image sizes in the datasets were different, because the float glasses produced by different manufacturers vary in width and thickness. Here, only the minimum resolution is presented in the above table.

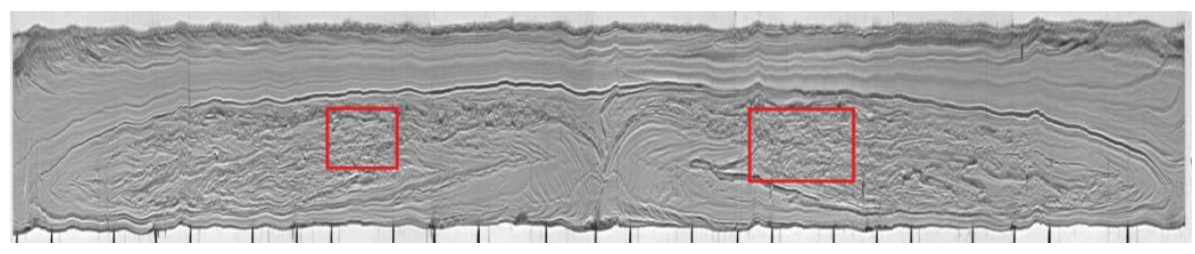

Figure 3. The flocculant defects

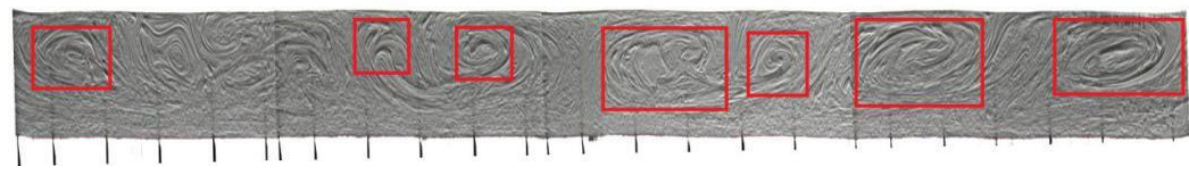

Figure 4. The curly defects

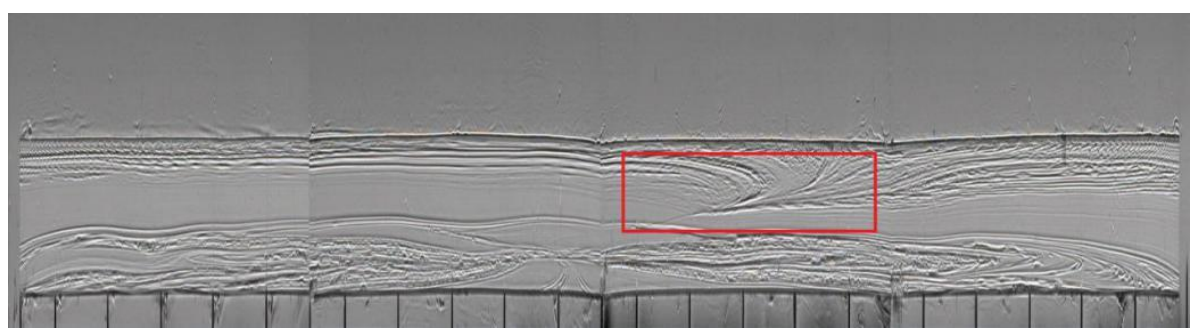

Figure 5. The hook-shaped defects 
In the experimental data, each image covers 1-3 kinds of defects, including 733 hook-shaped defects, 923 flocculent defects, and 812 curly defects (Figures 3-5). Different kinds of defects are distributed evenly. Some defect-free images were added to enhance the model generalizability.

\subsection{Software and hardware}

As shown in Table 3, the experimental framework was constructed on TensorFlow and Keras, programmed in Python, accelerated by graphic processing unit (GPU), and developed with PyCharm.

Table 3. The hardware and software of the experiments

\begin{tabular}{cc}
\hline Hardware & Software \\
\hline CPU: Intel Zeon & CentOS \\
Memory: 32GB & CUDA8.0 + CUDNN5.1 \\
GPU: Tensla V100 & Python3.6 + TensorFlow + Keras \\
\hline
\end{tabular}

\subsection{Results analysis}

Our model was trained under the TensorFlow+Keras framework in CentOS environment. The training was accelerated by Tesla V100GPU. Before initialization, the training parameters were pretrained by improved VGG16 under ImageNet.

\subsubsection{Influence of Laplacian convolutional layer}

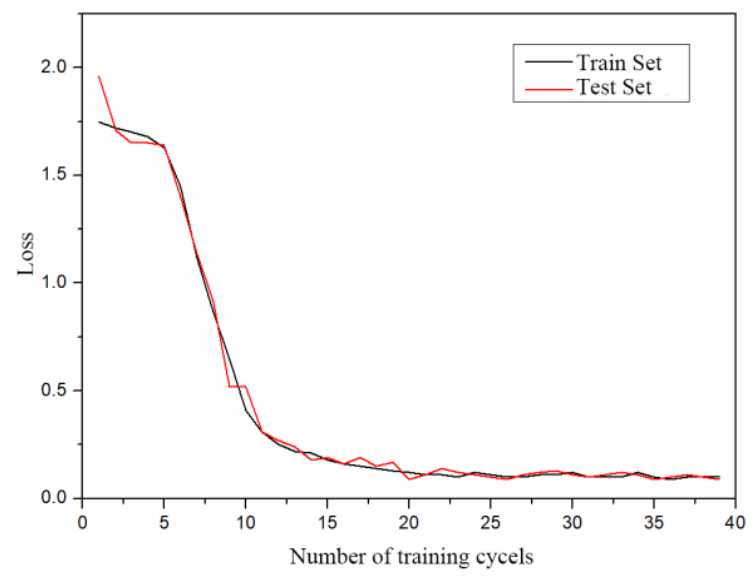

Figure 6. The loss curve after the addition of Laplacian convolutional layer

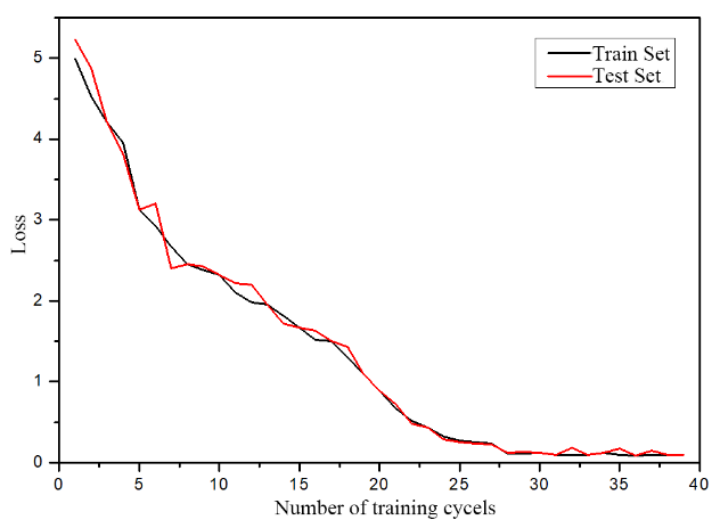

Figure 7. The loss curve before and after the addition of Laplacian convolutional layer
In this paper, a Laplacian convolutional layer is added to the original model. The parameters of this layer are fixed, without participating in training. The function of such parameters is to enhance the defect boundaries. Figures 6 and 7 compare the convergences of the original model and the model after adding the Laplacian convolutional layer. It can be seen that the addition of this layer sped up the convergence of the model: the model in Figure 6 converged after 20 cycles, while that in Figure 7 converged after 35 cycles.

\subsubsection{Influence of the improved VGG16 model}

In this paper, the feature extraction layer is improved from VGG16 model by replacing the last two fully connected layers were replaced with a fully connected layer of 1,024 nodes. The replacement simplifies the training parameters, and reduces the training time.

Table 4 compares the training times per cycle of the model before and after the replacement. Obviously, the improved VGG16 consumed 1/4 fewer time per cycle than the original VGG16.

Table 4. The training times per cycle of the model before and after the replacement

\begin{tabular}{ccc}
\hline & $\begin{array}{c}\text { Original } \\
\text { VGG16 }\end{array}$ & $\begin{array}{c}\text { Improved } \\
\text { VGG16 }\end{array}$ \\
\hline Training time per cycle & $5.23 \mathrm{mins}$ & $3.76 \mathrm{mins}$ \\
\hline
\end{tabular}

\subsubsection{Influence of anchor box resizing}

Considering the relatively large scale of end images, this paper increases the anchor box size of Faster RCNN, and eliminates the relatively small anchor boxes, highlighting the large defects features in end images which are closely correlated with the production process.

Table 5 compares the training times per cycle of the model before and after the resizing of anchor boxes. It can be seen that the fewer number of anchor boxes brought shorter training time and faster convergence.

Table 5. The training times per cycle of the model before and after anchor box resizing

\begin{tabular}{ccc}
\hline & Before resizing & After resizing \\
\hline Training time per cycle & $3.23 \mathrm{mins}$ & $2.76 \mathrm{mins}$ \\
\hline
\end{tabular}

\subsubsection{Actual detection effect}

During the test, the proposed glass defect detection model was applied on 400 test images, which contains 134 flocculant defects, 176 hook-shaped defects, and 121 curly defects.

Table 6 records the actual number, correctly detected number, missed number, and incorrectly detected number of different kinds of defects. The results show that our model detected $94 \%$ of the three different kinds of glass defects accurately, satisfying the requirements for actual production.

Figure 8 presents the test results of our model on actual glass. It can be seen that our model detected multiple flocculant defects and a hook-shaped defect. The flocculant defects spanned across the first of the glass flow layer, while the hookshaped defect resided in the third layer. The presence of flocculant defects in the first layer indicates that the raw materials are not fully melted in the production process. The operator should check whether the raw materials have ultrafine powder, and have rational participle size ratio. In addition, special attention should be paid to judge whether the calorific 
value of the fuel is sufficient, and whether the air-to-fuel ratio, and heat load distribution are reasonable. The existence of hook-shaped defect demonstrates the difference between production links in process conditions.

Table 6. The statistics on the detection of different kinds of defects

\begin{tabular}{cccccc}
\hline Type & Actual number & Correctly detected number & Missed number & Incorrectly detected number & Accurate \\
\hline Flocculant defect & 134 & 131 & 2 & 0 & $97 \%$ \\
Hook-shaped defect & 176 & 167 & 9 & 5 & $94 \%$ \\
Curly defect & 121 & 114 & 5 & 2 & $94 \%$ \\
\hline
\end{tabular}

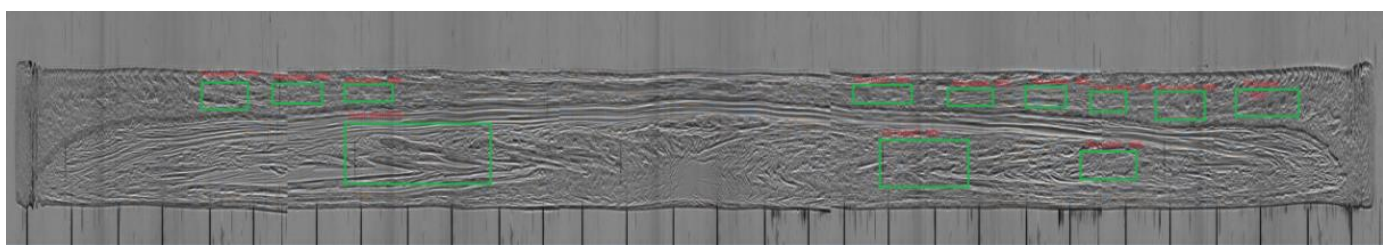

Figure 8. The glass defects actually detected by our model

\section{CONCLUSIONS}

(1) The deep learning was applied to detect the defects in end images of float glass, and set up a glass defect detection model.

(2) A Laplacian convolution layer was added to the target detection model Faster R-CNN, which improves the feature extraction layer, and the anchor box selection scheme was adjusted as per the features of glass defects.

(3) The actual production data were collected from large float glass manufacturers across China, and complied into a raw dataset with defect labels. Then, the proposed model was trained and tested on the prepared dataset. The test results show that our model achieved excellent performance on the test set, with a mean detection accuracy of $94 \%$.

\section{ACKNOWLEDGMENT}

This work was supported by the National Key Research and Development Program of China (Grant No.: 2016YFB0303700).

\section{REFERENCES}

[1] Feng, Z., Li, D., Qin, G., Liu, S. (2008). Study of the float glass melting process: combining fluid dynamics simulation and glass homogeneity inspection. Journal of the American Ceramic Society, 91(10): 3229-3234. https://doi.org/10.1111/j.1551-2916.2008.02606.x

[2] Feng, Z., Li, D., Qin, G., Liu, S. (2009). Effect of the flow pattern in a float glass furnace on glass quality: calculations and experimental evaluation of on - site samples. Journal of the American Ceramic Society, 92(12): 3098-3100. https://doi.org/10.1111/j.15512916.2009.03319.x

[3] Liu, S.M. (2010). Monitoring technology of the float glass side stripe. Journal of Wuhan University of Technology, 32(22): 101. https://doi.org/10.3963/j.issn.1671-4431.2010.22.024

[4] Ren, Y., Zhu, C., Xiao, S. (2018). Object detection based on fast/faster RCNN employing fully convolutional architectures. Mathematical Problems in Engineering, 2018: 1-7. https://doi.org/10.1155/2018/3598316
[5] Zhou, Q., Chen, R., Huang, B., Liu, C., Yu, J., Yu, X. (2019). An automatic surface defect inspection system for automobiles using machine vision methods. Sensors, 19(3): 644. https://doi.org/10.3390/s19030644

[6] Lei, X., Ouyang, H., Xu, L. (2019). Mature pomegranate recognition methods in natural environments using machine vision. Ciência Rural, 49(9). https://doi.org/10.1590/0103-8478cr20190298

[7] Zhang, X., Zhang, J., Ma, M., Chen, Z., Yue, S., He, T., $\mathrm{Xu}, \mathrm{X}$. (2018). A high precision quality inspection system for steel bars based on machine vision. Sensors, 18(8): 2732. https://doi.org/10.3390/s18082732

[8] El-Faki, M.S., Song, Y., Zhang, N., El-Shafie, H.A., Xin, P. (2018). Automated detection of parasitized Cadra cautella eggs by Trichogramma bourarachae using machine vision. International Journal of Agricultural and Biological Engineering, 11(3): 94-101. https://doi.org/10.25165/j.ijabe.20181103.2895

[9] Wang, Y.X., Xu, S.S., Li, W.B., Kang, F., Zheng, Y.J. (2017). Identification and location of grapevine sucker based on information fusion of 2D laser scanner and machine vision. International Journal of Agricultural and Biological Engineering, 10(2): 84-93. https://doi.org/10.3965/j.ijabe.20171002.2489

[10] Türkcan, S., Naczynski, D.J., Nolley, R., Sasportas, L.S., Peehl, D.M., Pratx, G. (2016). Endoscopic detection of cancer with lensless radioluminescence imaging and machine vision. Scientific Reports, 6: 30737. https://doi.org/10.1038/srep30737

[11] Xiong, H.L., Fan, C.Q., Zhao, S., Yu, Y. (2019). Detection method of glass surface defects based on multi-scale convolution neural network. Computer Integrated Manufacturing Systems, 2019: 1-16. http://kns.cnki.net/kcms/detail/1 1.5946.tp.20190708.11 37.014.html.

[12] Yao, Z.H. (2018). Discussion on online detection technology of glass production defects. Fujian Chemical Industry, 2018(5): 264-265, 271.

[13] Li, C.Y., Liu, Z., Li, S.T. (2018). Low-contrast surface defect detection algorithm for flat glass. Mechanical Engineer, 2018(3): 21-23. https://doi.org/10.3969/j.issn.1002-2333.2018.03.008

[14] Luo, C., Gao, J., Sha, F.Y., Luo, F. (2016). Research on on-line defect detection system based on machine vision. Digital Technology and Application, 2016(4): 46-48. 
[15] Girshick, R., Donahue, J., Darrell, T., Malik, J. (2014). Rich feature hierarchies for accurate object detection and semantic segmentation. In Proceedings of the IEEE Conference on Computer Vision and Pattern Recognition, 32(11): 580-587.

[16] Li, J., Liang, X., Shen, S., Xu, T., Feng, J., Yan, S. (2017). Scale-aware fast R-CNN for pedestrian detection. IEEE transactions on Multimedia, 20(4): 985-996. https://doi.org/10.1109/TMM.2017.2759508

[17] Ren, S., He, K., Girshick, R., Sun, J. (2015). Faster RCNN: Towards real-time object detection with region proposal networks. In Advances in Neural Information Processing Systems, 91-99.

[18] Liu, W., Anguelov, D., Erhan, D., Szegedy, C., Reed, S., Fu, C.Y., Berg, A.C. (2016). Ssd: Single shot multibox detector. In European Conference on Computer Vision, 9905: 21-37. https://doi.org/10.1007/978-3-319-46448$0 \_2$

[19] Redmon, J., Divvala, S., Girshick, R., Farhadi, A. (2016). You only look once: Unified, real-time object detection. In Proceedings of the IEEE Conference on Computer Vision and Pattern Recognition, pp. 779-788.

[20] Cholakkal, H., Johnson, J., Rajan, D. (2018). Backtracking spatial pyramid pooling-based image classifier for weakly supervised top-down salient object detection. IEEE Transactions on Image Processing,
27(12):

6064-6078.

https://doi.org/10.1109/TIP.2018.2864891

[21] Tang, Y., Zheng, L., Ren, F., Zhu, H., Lin, Z. (2007). Study on automatic location for interested region of image based on geometrical features. Jisuanji Gongcheng/ Computer Engineering, 33(1): 200-203.

[22] Yang, Q., Cheng, M., Zhou, W., Chen, Y., Qiu, M., Lin, W., Chu, W. (2018). Inceptext: A new inception-text module with deformable psroi pooling for multi-oriented scene text detection. arXiv preprint arXiv:1805.01167.

[23] Vu, T., Jang, H., Pham, T.X., Yoo, C. (2019). Cascade RPN: Delving into high-quality region proposal network with adaptive convolution. In Advances in Neural Information Processing Systems, 1432-1442.

[24] Omar, A. (2019). Lung CT Parenchyma Segmentation using VGG-16 based SegNet Model. In IJCA, 178(44): 10-13.

[25] Merris, R. (1994). Laplacian matrices of graphs: a survey. Linear Algebra and Its Applications, s197-198(2): 143176.

[26] Chen, Y., Li, W., Sakaridis, C., Dai, D., Van Gool, L. (2018). Domain adaptive faster R-CNN for object detection in the wild. In Proceedings of the IEEE Conference on Computer Vision and Pattern Recognition, 3339-3348. 